\title{
Vernalização afeta a mobilização de reservas de açúcares e nitrogênio e a emergência de plantas de lírio (Lilium longiflorum thunb.) 'snow queen'1
}

\author{
Vernalization affects nitrogen and sugar mobilization and plant emergence in 'snow queen' lily \\ (Lilium longiflorum thunb.)
}

\author{
Claudia Nardi ${ }^{2}$ Galileo Adeli Buriol ${ }^{3}$ Rogério Antônio Bellé ${ }^{4}$ \\ Nereu Augusto Streck ${ }^{5}$ Mariângela Schuh ${ }^{6}$
}

\section{RESUMO}

Com o objetivo de avaliar a resposta dos dias efetivos de vernalização do lírio sobre o conteúdo de açúcares solúveis e nitrogênio total e a emergência de plantas, bulbos de Lilium longiflorum, $c v$ 'Snow Queen' foram submetidos a diferentes temperaturas de vernalização e períodos de exposição à temperaturas vernalizantes e cultivados em estufa plástica. O experimento foi conduzido na Universidade Federal de Santa Maria, Santa Maria (RS), em duas épocas de plantio dos bulbos: de 20/03/02 a 05/11/02 e 05/12/02 a 15/05/03. Os tratamentos foram 0, 9, 11, 14, 19, 23, 28, 29, $34,38,42,45$ e 56 dias efetivos de vernalização (DV), calculados a partir de uma função beta de resposta da vernalização à temperatura. Determinaram-se o conteúdo de açúcares solúveis e nitrogênio total e a duração do subperíodo de plantio dos bulbos-emergência das plantas $(P-E)$. A vernalização promoveu a mobilização de reservas de açúcares e nitrogênio, o que é importante para nutrição da planta em emergência. O aumento do número de dias efetivos de vernalização reduziu a duração do subperíodo $P$ E. Bulbos tratados em temperaturas de $10^{\circ} \mathrm{C}$ por 6 e 8 semanas de armazenamento (34 e 45DV), apresentaram uma menor duração do subperíodo P-E.

Palavras-chave: vernalização, temperatura, Lilium, dias efetivos de vernalização.

\section{ABSTRACT}

In order to evaluate the response of effective vernalization days on the soluble sugar and total nitrogen contents and the emergence of Lilium longiflorum plant shoots, cv 'Snow Queen' bulbs were kept under different vernalization temperatures and periods of treatment under

\begin{abstract}
vernalizing temperatures, and cultivated in a plastic greenhouse after the vernalization. The experiment was carried out at the Universidade Federal de Santa Maria, RS, in two seasons: from 03/20/02 to 11/05/02 and from 12/05/02 to 05/ 15/03. The treatments were 0, 9, 11, 14, 19, 23, 28, 29, 34, $38,42,45$ and 56 effective vernalization days, calculated with a beta response function to temperature. The soluble sugar and total nitrogen contents, and the duration of the planting-emergence $(P-M)$ phase were determined. The vernalization promoted the mobilization of sugar and nitrogen reserves, which is important for the nutrition of the emergence of the shoot. The increasing number of the effective vernalization days reduced the duration of the $P$ - $E$ phase. Bulbs treated under temperatures of $10^{\circ} \mathrm{C}$ for 6 to 8 weeks of storage (34 and $45 \mathrm{DV}$ ), had the shortest duration of the P-E phase.
\end{abstract}

Key words: vernalization, temperature, Lilium, effective vernalization days.

\section{INTRODUÇÃO}

O lírio (Lilium longiflorum Thunb.) é uma espécie que requer a exposição dos bulbos a baixas temperaturas, para acelerar a emergência e o consequente florescimento das plantas, processo esse chamado de vernalização (CHOUARD, 1960). Os trabalhos de PURVIS (1939, 1947, 1948) e PURVIS \& GREGORY (1937, 1952) são as bases dos atuais conhecimentos sobre a vernalização em plantas. Desde então, têm sido muito estudados os efeitos das temperaturas de vernalização, tais como as faixas de

\footnotetext{
${ }^{1}$ Parte da Tese de Doutorado apresentada pelo primeiro autor ao Programa de Pós-graduação em Agronomia, Centro de Ciências Rurais (CCR), Universidade Federal de Santa Maria (UFSM).

2Engenheiro Agrônomo, Doutor em Agronomia. E-mail: claudianardi@ hotmail.com

${ }^{3}$ Engenheiro Agrônomo, Doutor, Professor Aposentado, Departamento de Fitotecnia (DF), CCR, UFSM, 97105-900, Santa Maria,RS. Bolsista do Conselho Nacional de Desenvolvimento Tecnológico ( $\mathrm{CNPq})$. Autor para correspondência.

${ }^{4}$ Engenheiro Agrônomo, Doutor, Professor Adjunto, DF, CCR, UFSM.

${ }^{5}$ Engenheiro Agrônomo, PhD, Professor Ajunto, DF, CCR, UFSM.

${ }^{6}$ Aluno do Curso de Graduação em Agronomia, CCR, UFSM.
} 
temperaturas nas quais o processo ocorre, duração do período de exposição a temperaturas vernalizantes, respostas genotípicas e a idade da planta (CHUJO, 1996; JEDEL et al., 1986; WANG et al., 1995; RAWSON et al., 1998). A eficiência da vernalização depende da combinação de dois fatores: a temperatura e a duração do período de exposição (YAN \& HUNT, 1999), sendo a duração do período de exposição a temperaturas vernalizantes expressa em dias efetivos de vernalização. Um dia efetivo de vernalização é obtido quando a planta é submetida à temperatura ótima de vernalização por 24 horas (STRECK, 2002b, 2003).

A vernalização do bulbo acelera a emergência e o florescimento da planta na maioria das espécies de lírio, particularmente no lírio branco, Lilium longiflorum (WANG \& ROBERTS, 1970; WEILER, 1992; LEE et al., 1996). Entretanto, a temperatura ótima de vernalização e a duração mínima do tratamento de vernalização diferem entre espécies e entre cultivares (ROH \& WILKINS, 1977; WEILER \& LANGHANS, 1968, LIN \& WILKINS, 1975). O tempo para emergência da planta de lírio e o decréscimo na variabilidade do tempo de emergência em uma população estão associados à duração do período de vernalização (MILLER \& KIPLINGER, 1966; WEILER \& LANGHANS, 1968; ERWIN \& EIGLES, 1998).

SegundoMOE \& WICKSTROM $(1973,1979)$, o frio afeta principalmente a conversão de amido em açúcares solúveis para o suprimento de carboidratos, induzindo uma rápida brotação do bulbo, reduzindo assim o período brotação-floração. A vernalização promove a despolimerização dos carboidratos, resultando em um aumento dos açúcares solúveis e dos conteúdos de nitrogênio solúveis (CHOUARD, 1960). REES (1966) também sugere que baixas temperaturas agem promovendo a conversão do amido armazenado em açúcares solúveis. O acúmulo de açúcares solúveis e glutamina nas escamas dos bulbos pode promover o rápido crescimento de folhas e haste e o florescimento normal durante o cultivo (ZACHARIUS etal. 1957; OHYAMAet al.,1988).

OHYAMA et al. (1988) observaram em tulipas (espécie semelhante ao lírio em termos de exigências em vernalização) vernalizadas a $5^{\circ} \mathrm{C}$ por um período de dois meses, que ocorreu uma rápida degradação do amido após o início do tratamento e um considerável acúmulo de sacarose simultaneamente à degradação. Nos bulbos nãovernalizados, ao contrário, a conversão do amido a açúcares solúveis não ocorreu antes do plantio.

Em experimentos conduzidos por WANG \& ROBERTS (1970), com a cultivar Ace de L. longiflorum, vernalizada a $4,4^{\circ} \mathrm{C}$ por $0,3,6$ e 9 semanas, concluíram que os dias para emergência do bulbo diminuiram em função do tempo de armazenamento. As concentrações de N-protéico e aminoácidos livres aumentaram com o tempo de armazenamento a $4,4^{\circ} \mathrm{C}$. O armazenamento do bulbo por seis semanas a $4,4^{\circ} \mathrm{C}$ foi efetivo na superação da dormência, o que refletiu na redução do tempo de emergência do broto, o qual foi apenas 37 dias comparados a 80 dias para bulbos não-vernalizados. Segundo esses autores, o armazenamento a $4,4^{\circ} \mathrm{C}$, tratamento com $\mathrm{GA}_{3}$ e temperaturas do solo elevadas no campo, podem estar associados à redução no conteúdo de inibidores, o que conduz à superação da dormência.

O presente trabalho teve como objetivos quantificar o conteúdo de açúcares solúveis e nitrogênio total, e a duração do subperíodo "plantio dos bulbos-emergência das plantas" oriundas de bulbos não-vernalizados e de bulbos submetidos a diferentes temperaturas e períodos de vernalização em duas épocas de cultivo.

\section{MATERIALEMÉTODOS}

O trabalho foi conduzido no Campo Experimental do Departamento de Fitotecnia - Polo de Difusão da Plasticultura, da Universidade Federal de Santa Maria, RS em estufa plástica. Foram realizadas duas épocas de plantio com a cultivar "Snow Queen" de lírio Híbrido Longiflorum. A primeira época foi de 20/03/02 a 05/11/02 e a segunda época de 05/12/02 a $15 / 05 / 03$. O delineamento experimental foi o de blocos ao acaso com três repetições e os tratamentos foram dias efetivos de vernalização (DV).

Para o cálculo dos dias efetivos de vernalização, a taxa diária de vernalização [ $f v n(T)$, dia $^{-1}$ ] foi calculada com uma função beta (STRECK, 2002a, 2003). A função beta apresenta três coeficientes, que são as temperaturas cardinais $\left(\mathrm{T}_{\text {min }}, \mathrm{T}_{\text {ótima }}, \mathrm{e}_{\text {máx }}\right)$, e é definida como:

$$
\begin{aligned}
& \text { fvn }(\mathrm{T})=\left[2\left(\mathrm{~T}_{\mathrm{min}} \mathrm{T}^{\alpha}\left(\mathrm{T}_{\text {ótima }}-\mathrm{T}_{\min }\right)^{\alpha}-\left(\mathrm{T}-\mathrm{T}_{\min }\right)^{2 \alpha}\right] /\left(\mathrm{T}_{\text {ótima }}-\mathrm{T}_{\min }\right)^{2 \alpha}(1)\right. \\
& \alpha=\ln 2 / \ln \left[\left(\mathrm{T}_{\max }-\mathrm{T}_{\text {min }}\right) /\left(\mathrm{T}_{\text {ótima }}-\mathrm{T}_{\text {min }}\right)\right]
\end{aligned}
$$

em que $\mathrm{T}_{\text {min }}$ corresponde à temperatura mínima, $\mathrm{T}_{\text {ótima }}$, a temperatura ótima e $\mathrm{T}_{\text {máx }}$ a temperatura máxima, respectivamente. Assumiu-se que as temperaturas cardinais de vernalização do lírio são $-1,5^{\circ} \mathrm{C}$ (MCRAE, 1998; LEE \& ROH, 2001), $4^{\circ} \mathrm{C}$ (HEINS et al., 1982; MILLER \& LANGHANS, 1989; LEE et al., 1996; WANG \& ROBERTS, 1970) e $21^{\circ} \mathrm{C}$ (MILLER \& KIPLINGER, 1966; WEILER \& LANGHANS, 1968). Com estas pressuposições, a reposta da função beta (Equações 1 e 2) é apresentada na figura 1 . 


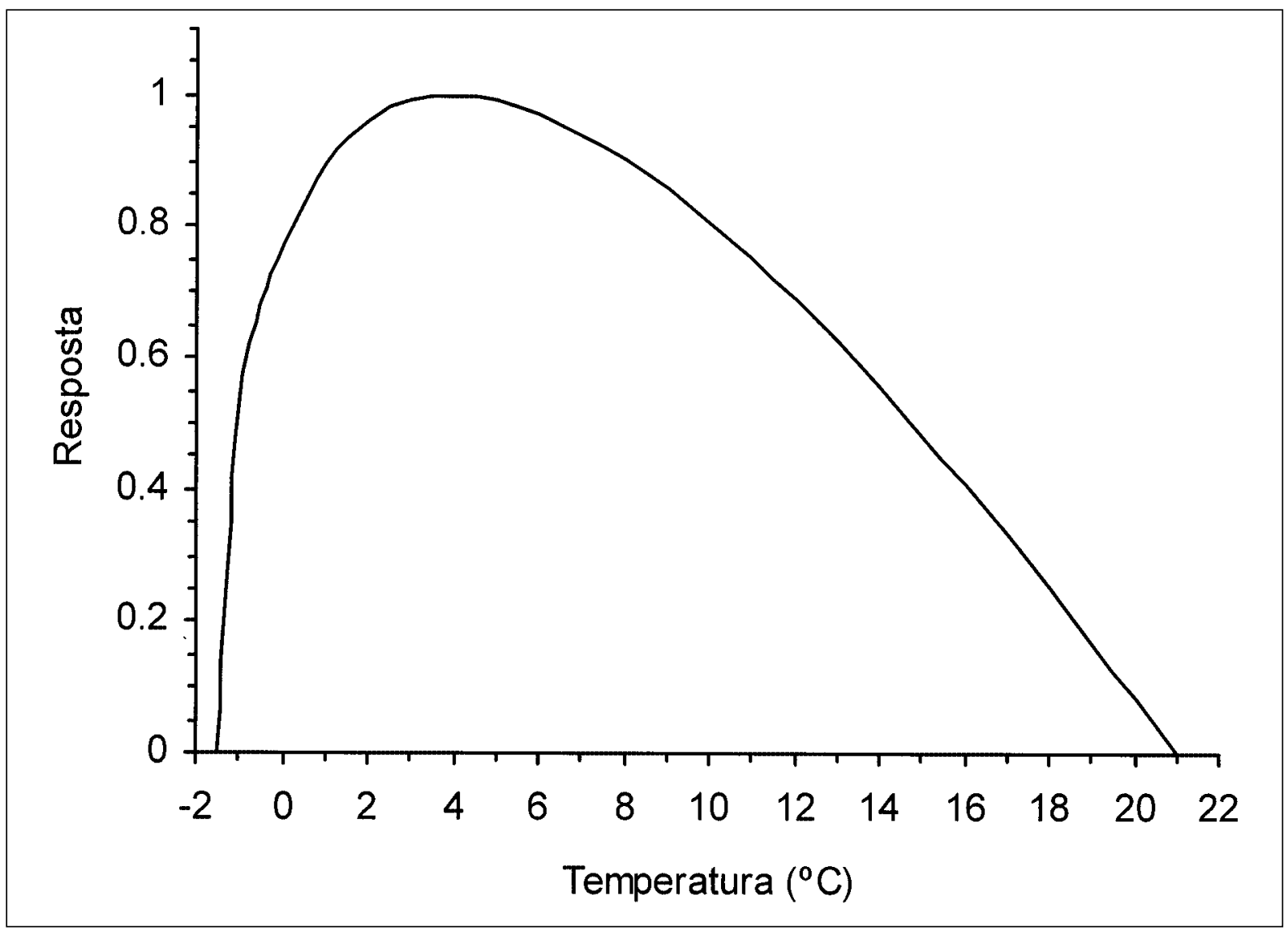

Figura 1 - Função beta usada para descrever a taxa diária de vernalização $[\mathrm{fvn}(\mathrm{T})]$ em Lilium longiflorum com $\mathrm{T}_{\min }=-1,5^{\circ} \mathrm{C}, \mathrm{T}_{\text {бtima }}=4^{\circ} \mathrm{C}$ e $\mathrm{T}_{\text {máx }}=21^{\circ} \mathrm{C}$.

A duração da exposição às temperaturas de vernalização é medida por dias efetivos de vernalização (DV). A partir da temperatura ótima de vernalização somente uma fração de DV é acumulado pela planta durante um dia do calendário oficial.

Os dias efetivos de vernalização, DV, foram calculados pela equação:

$\mathrm{DV}=\mathrm{Sfvn}(\mathrm{T})$

em que fvn(T) é a taxa diária de vernalização $\left(\operatorname{dia}^{-1}\right)$, calculada com as equações (1) e (2). A partir das temperaturas de $-0,5^{\circ} \mathrm{C} ; 4^{\circ} \mathrm{C}, 10^{\circ} \mathrm{C}$, sem vernalizar e das durações de 2, 4, 6 e 8 semanas de armazenamento estimavam-se os dias efetivos de vernalização, resultando nos 13 tratamentos listados na tabela 1.

Os bulbos foram vernalizados no Núcleo de Pós-colheita do Departamento de Fitotecnia/ CCR/UFSM. Na primeira época de plantio, todos os bulbos entraram em vernalização no dia 06 de março de 2002, e após duas semanas, no dia 20/03/02 foram retiradas amostras dos bulbos armazenados na câmara, e sem vernalizar e plantados na estufa. $\mathrm{O}$
Tabela 1 - Dias efetivos de vernalização (DV) correpondentes as temperaturas de vernalização e durações dos períodos de armazenamento, para cultura do lírio, cultivar Snow Queen. Santa Maria, RS, 2003.

\begin{tabular}{cl}
\hline $\begin{array}{c}\text { Dias efetivos de } \\
\text { vernalização(DV) }\end{array}$ & \multicolumn{1}{c}{$\begin{array}{c}\text { Temperaturas } x \text { Semanas de } \\
\text { Armazenamento }\end{array}$} \\
\hline $9 \mathrm{DV}$ & $-0,5^{\circ} \mathrm{C}$ por 2 semanas \\
$19 \mathrm{DV}$ & $-0,5^{\circ} \mathrm{C}$ por 4 semanas \\
$29 \mathrm{DV}$ & $-0,5^{\circ} \mathrm{C}$ por 6 semanas \\
$38 \mathrm{DV}$ & $-0,5^{\circ} \mathrm{C}$ por 8 semanas \\
$14 \mathrm{DV}$ & $4^{\circ} \mathrm{C}$ por 2 semanas \\
$28 \mathrm{DV}$ & $4^{\circ} \mathrm{C}$ por 4 semanas \\
$42 \mathrm{DV}$ & $4^{\circ} \mathrm{C}$ por 6 semanas \\
$56 \mathrm{DV}$ & $4^{\circ} \mathrm{C}$ por 8 semanas \\
$11 \mathrm{DV}$ & $10^{\circ} \mathrm{C}$ por 2 semanas \\
$23 \mathrm{DV}$ & $10^{\circ} \mathrm{C}$ por 4 semanas \\
$34 \mathrm{DV}$ & $10^{\circ} \mathrm{C}$ por 6 semanas \\
$45 \mathrm{DV}$ & $10^{\circ}$ por 8 semanas \\
$0 \mathrm{DV}$ & Sem vernalizar \\
\hline
\end{tabular}

Ciência Rural, v. 34, n.4, jul-ago, 2004. 
mesmo procedimento foi realizado em 03/04/02 (bulbos de 4 semanas); em 17/04/02 (bulbos de 6 semanas) e em 01/05/02 (bulbos que estavam armazenados a 8 semanas). E, na segunda época de plantio os bulbos entraram em vernalização no dia 10/10/02 (bulbos de 8 semanas), 24/10/02 (bulbos de 6 semanas), 07/11/02 (bulbos de 4 semanas) e 21/ 11/02 (bulbos de 2 semanas). Os bulbos de todos os tratamentos foram retirados da câmara e plantados no mesmo dia, em 05/12/02, sendo o processo utilizado nesta época o inverso do realizado na primeira época de plantio.

A hipótese de uma provável variação nos conteúdos de nitrogênio e açúcares de bulbos que não estavam submetidos a temperaturas vernalizantes, conduziram ao acondicionamento dos bulbos de todos os tratamentos na câmara em 20/03/02, logo após a colheita desses bulbos, os quais foram retirados da câmara em datas diferentes na primeira época. Essa metodologia visou evitar que os processos metabólicos do interior do bulbo, que poderiam estar acontecendo durante a sua permanência fora da câmara mascarasse as respostas à vernalização. Isso favoreceu as determinações dos açúcares e nitrogênio, porém, desfavoreceu as determinações das variáveis obtidas em nível de campo, devido à temperatura e ao fotoperíodo serem diferentes na data de plantio. Desse modo, o tratamento de 8 semanas teve seu plantio 42 dias após os bulbos de duas semanas. Portanto, na segunda época o processo usado foi o inverso, no qual os bulbos entraram na câmara em datas diferentes, mas todos foram plantados no campo no mesmo dia.

As análises de nitrogênio total e açúcares solúveis foram realizadas no Laboratório de Análises Físico-químicas do Departamento de Tecnologia e Ciência dos Alimentos/CCR/UFSM. A determinação do nitrogênio total foi realizada pelo processo de digestão Kjeldahl conforme metodologia descrita pela AOAC International (1998) e a de açúcares redutores em glicose e não redutores em sacarose para a estimativa dos açúcares solúveis segundo as Normas Analíticas do Instituto Adolfo Lutz (1985).

As determinações de açúcares solúveis e nitrogênio total na primeira época foram realizadas no momento da retirada dos bulbos da câmara e sem vernalizar para o plantio: 20/03/02, 03/04/02, 17/04/02 e 01/05/02 e, na segunda época no dia 10/10/02, quando os bulbos entraram na câmara para vernalização, ou seja, bulbos que seriam mantidos por 8 semanas, e no dia 07/11/02 nos bulbos que seriam mantidos por 4 semanas. No dia 05/12/02, as determinações foram feitas nas amostras de bulbos de todos os tratamentos que foram mantidos na câmara $\left(-0,5^{\circ} \mathrm{C}, 4^{\circ} \mathrm{C}, 10^{\circ} \mathrm{C}\right.$ por 2 ,
4, 6 e 8 semanas, mais o tratamento sem vernalização) e que estavam sendo levados a campo. Estas análises de bulbos sem vernalizar, antes da entrada na câmara em 10/10/02 e 07/11/02, e depois na retirada dos bulbos para o plantio, teve objetivo de quantificar a variação do nitrogênio total e açúcares solúveis dos bulbos até o momento do plantio.

O número de dias para emergência da planta foi determinado pela contagem diária das plantas emergidas até observar 50\% de emergência. A análise estatística constou do uso da análise de regressão, selecionando-se o modelo matemático que melhor explicasse o comportamento das variáveis do ponto de vista biológico.

\section{RESULTADOS E DISCUSSÃO}

O dados obtidos de bulbos nãovernalizados evidenciaram alterações nos conteúdos de nitrogênio total e açúcares solúveis nas amostras das duas épocas (Figura 2). Estes resultados indicam que, quanto mais tempo estes bulbos permanecerem armazenados, mesmo não estando expostos a temperaturas vernalizantes, mais intensas serão as mobilizações de reservas. Estes resultados discordam daqueles encontrados por OHYAMA et al. (1998), em que bulbos não-vernalizados não apresentaram a conversão de amido a açúcares solúveis antes do plantio.

Para os resultados do nitrogênio total em função dos dias efetivos de vernalização, observa-se uma variação semelhante nos bulbos das duas épocas, mostrando que há uma tendência de redução dessa mobilização com o aumento do número de dias efetivos de vernalização (Figura 3a). Estes resultados apresentam algumas similaridades com aqueles obtidos por WANG \& ROBERTS (1970) com a cultivar Ace de L. longiflorum, nos quais o N-protéico aumentou com o tempo de armazenamento, e ainda corroboram com as observações de CHOUARD (1960), em que a vernalização promove a despolimerização de reservas de proteínas, resultando em um aumento de compostos de nitrogênio solúveis. Embora os valores de $\mathrm{r}^{2}$ sejam baixos (Figura 3a), a seleção de uma equação de terceiro grau, que aumentou bastante o $\mathrm{r}^{2}$ (acima de $0,95)$ não foi adotada por não apresentar interpretação biológica para estas variáveis.

Os açúcares solúveis apresentaram variações diferentes entre os bulbos usados na primeira e segunda época de plantio (Figura 3b), porém, nas duas épocas, as maiores concentrações foram registradas nos tratamentos que apresentaram um maior número de dias efetivos de vernalização. Estes resultados confirmam as observações 


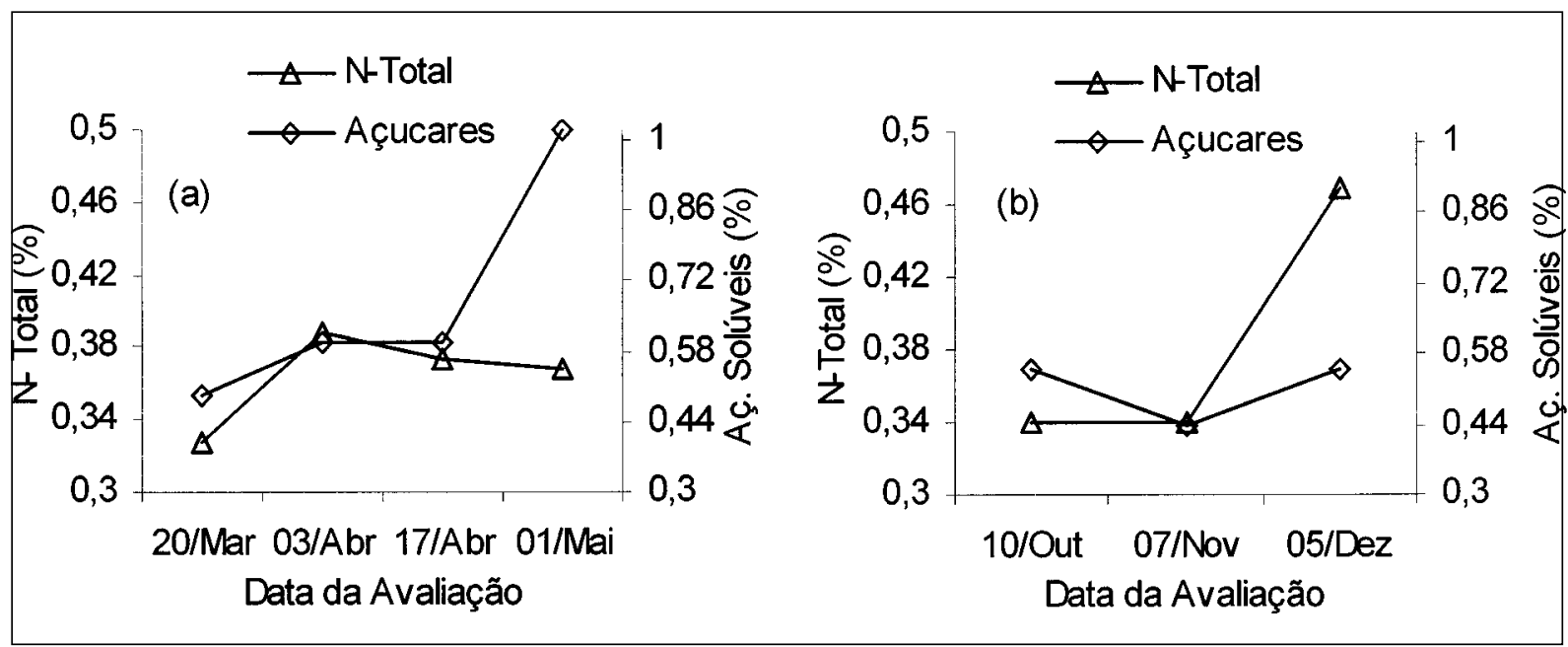

Figura 2 - Nitrogênio total e açúcares solúveis em bulbos do lírio 'Snow Queen' não-vernalizados, utilizados na primeira (a) e segunda (b) épocas de plantio. Santa Maria, 2003.

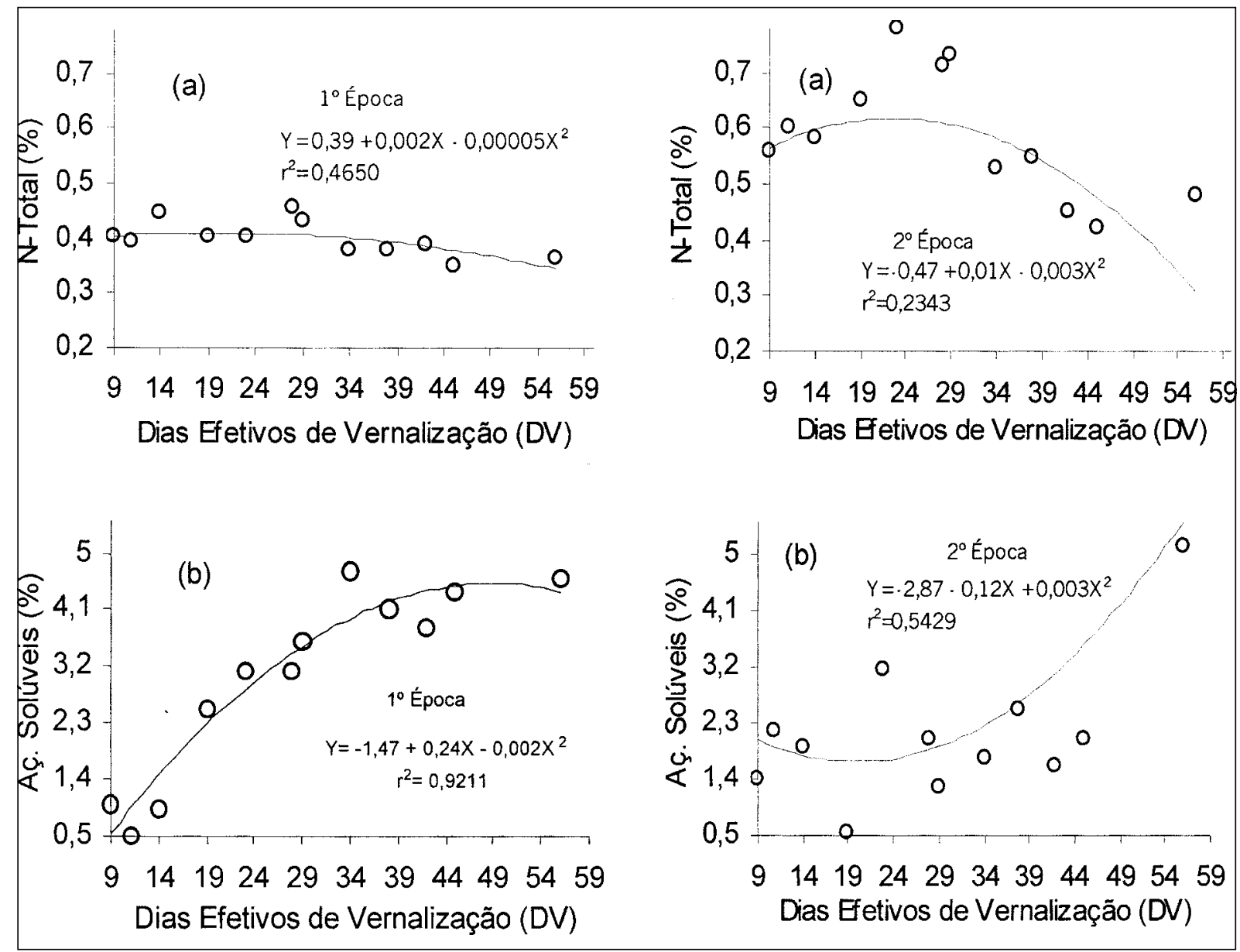

Figura 3 - Nitrogênio total (a) e açúcares solúveis (b) em bulbos do lírio 'Snow Queen' para diferentes dias efetivos de vernalização, utilizados no cultivo na primeira e segunda épocas. Santa Maria, 2003.

Ciência Rural, v. 34, n.4, jul-ago, 2004. 
feitas por OHYAMA(1988), CHOUARD (1960), MOE \& WICKSTROM (1973, 1979), REES (1966), que o frio promove a mobilização de reservas de amido a açúcares solúveis e subseqüente acúmulo desses constituintes.

A duração do subperíodo plantioemergência dos bulbos não-vernalizados na primeira época de plantio, apresentaram uma redução linear decrescente, quando plantados após o armazenamento de 2, 4, 6, 8 (Figura 4a). Na segunda época, os bulbos não-vernalizados ficaram armazenados por oito semanas e foram plantados em uma única data (05/12/ 02) e levaram em média 90 dias para atingir $50 \%$ de emergência, período esse bastante superior aos bulbos não-vernalizados na primeira época, independente da data do plantio.

Para os bulbos vernalizados, a duração do subperíodo plantio-emergência das plantas diminuiu com o aumento do número de dias efetivos de vernalização (Figura 4b). Estes resultados estão de acordo com os resultados reportados em MILLER \& KIPLINGER, (1966); WEILER \& LANGHANS, (1968); ROH \& WILKINS, (1977a); LIN \& WILKINS, (1975); LEE et al. (1996), WANG \& ROBERTS (1970) e ERWIN \& EIGLES (1998). Essa redução da duração do subperíodo plantio-emergência com o aumento de DV pode estar relacionada com a mobilização das reservas para o rápido crescimento do broto conforme proposto por MOE \& WICKSTROM $(1973,1979)$ tendo em vista que os resultados de açúcares solúveis e nitrogênio total (Figuras 3a e b) mostram essa tendência.

$\mathrm{Na}$ segunda época de plantio, não foi registrado efeito dos tratamentos dos dias efetivos de vernalização na emergência das plantas, possivelmente devido às temperaturas do solo bastante elevadas nesse período, o que contribuiu para a superação da dormência do bulbos que se encontravam parcialmente vernalizados.

Os resultados deste trabalho mostraram que a vernalização do bulbo acelera a emergência da planta. Na produção comercial de lírio, a vernalização é muito importante não somente por acelerar a emergência da planta, mas por promover a indução floral e reduzir o ciclo da cultura.

\section{CONCLUSÕES}

A vernalização promove a mobilização de reservas de açúcares e nitrogênio, o que é importante para nutrição da planta em emergência. $\mathrm{O}$ aumento do número de dias efetivos de vernalização reduziu a duração do subperíodo P-E. Bulbos tratados a temperaturas de $10^{\circ} \mathrm{C}$ por 6 e 8 semanas de armazenamento ( 34 e $45 \mathrm{DV}$ ), apresentaram uma menor duração do subperíodo plantio dos bulbos emergência das plantas.

\section{REFERÊNCIAS BIBLIOGRÁFICAS}

CHOUARD, P. Vernalization and its relations to dormancy. Annual Review of Plant Physiology, v.11, n.1, p.191-238, 1960.

CHUJO, H. Differences in vernalization effects in wheat under various temperatures. Proceedings of the Crop Science Society of Japan, v. 35, n.1, p. 235-242, 1996.

ERWIN, J.E.; EIGLES, G.E. Influence of simulated shipping and rooting temperature and production year on easter lily (Lilium longiflorum Thunb.) development. Journal of the American Society for Horticultural Science, v.123, n.2, p. 230-233, 1998 .
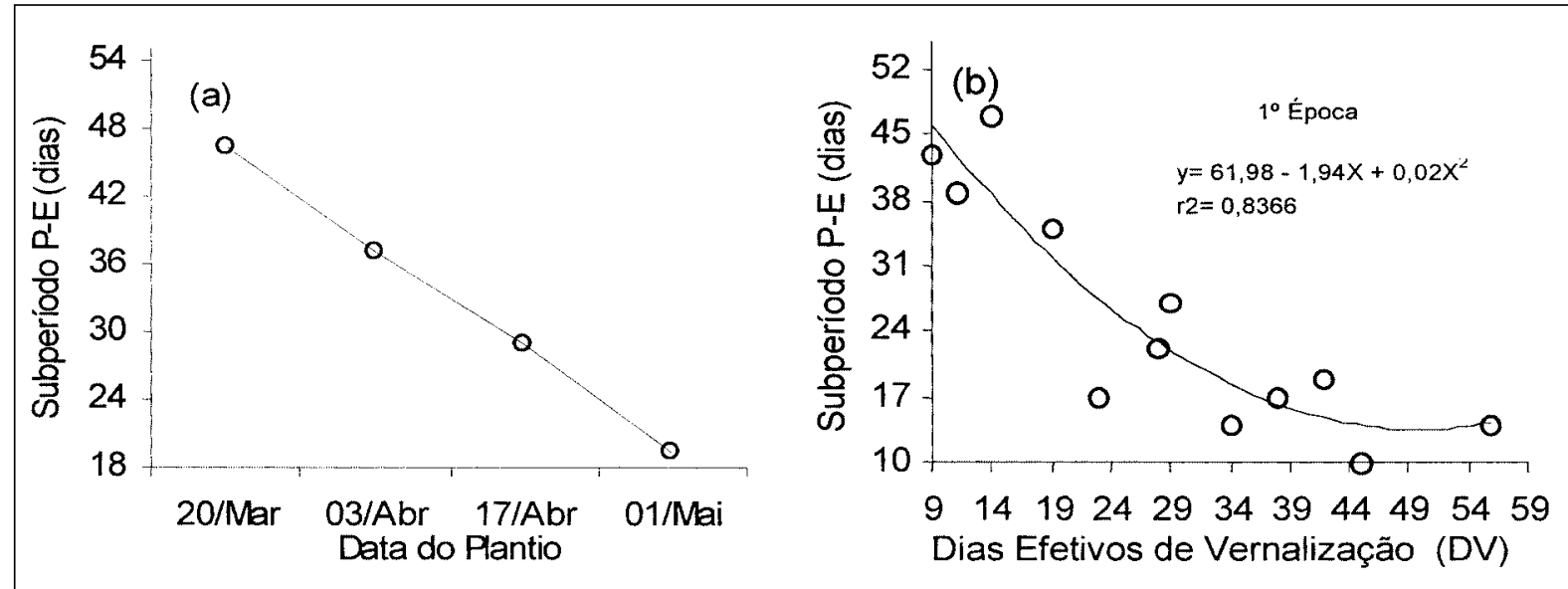

Figura 4 - Duração do subperíodo plantio-emergência de plantas provenientes de bulbos não-vernalizados do lírio 'Snow Queen' na primeira época de plantio (a) e, duração do subperíodo plantio-emergência de plantas provenientes de bulbos com diferentes dias efetivos de vernalização, na primeira época de plantio (b). Santa Maria, 2003. 
HEINS, R.D.; PEMBERTIN, H.B.; WILKINS, H.F. The influence of light on lily (Lilium longiflorum Thunb.). I. Influence of light intensity on plant development. Journal of the American Society for Horticultural Science, v.107, n.3, p.330-335, 1982.

JEDEL, P.E.; EVANS, L.E.; SCARTH, R. Vernalization responses of a celected group of spring wheat (Triticum aestivym L.) cultivars. Canadian Journal of Plant Science, v.66, n.1, p.1-9, 1986.

LEE, S.J.; ROH, M.S. Influence of frozen storage duration and forcing temperature on flowering of oriental hybrid lilies. HortScience, v.36, n.6, p.1053-1056, 2001.

LEE, S.J.; KIM, Y.A.; WANG, H.J. Effect of bulb vernalization on the growth and flowering of asiatic hybrid lily. Acta Horticulture, v.414, p.229-234, 1996.

LIN, W.C.; WILKINS, H.F. Influence of bulb harvest date and temperature on growth and flowering of Lilium longiflorum. Journal of the American Society for Horticultural Science, v.100, n.1, p.6-9, 1975

McRAE, E.A. Lilies: a guide for growers and collectors. Portland : Timber, 1998. 392p.

MILLER, W.B.; LANGHANS, R.W. Reduced irradiance affects dry weight partitioning in easter lily. Journal of the American Society for Horticultural Science, v.114, n.102, p.306-309, 1989.

MILLER, W.B.; KIPLINGER, D.C. Interaction of temperatures and time of vernalization on northwest easter lilies. Proceedings of the American Society for Horticultural Science, v.88, p.335-345, 1966.

MOE, R.; WICKSTROM, A. The effect of storage temperature on shoot growth, flowering, and carbohydrate metabolism in tulip tulbs. Physiologia Plantarum, v.28, p.81-87, 1973.

Effect of precoolong at 5 or $-1^{\circ} \mathrm{C}$ on shoot growth, flowering, and carbohydrate metabolism in tulip bulbs. Scientia Horticulturae, v.10, p.187-201, 1979.

NORMAS ANALÍTICAS DO INSTITUTO ADOLFO LUTZ. 3.ed. São Paulo : O Instituto, 1985. 533p.

OFFICIAL METHODS OF ANALYSIS OF AOAC INTERNACIONAL. Edited by Patricia Cunniff - 16.ed. 4.rev. Maryland : AOAC International, 1998. 2v.

OHYAMA, T.; IKARASHI, T.; BABA, A. Effect of cold storage treatment for forcing bulbs on the $\mathrm{C}$ and $\mathrm{N}$ metabolism of tulip plants. Soil Science and Plant Nutrition, v.34, n.4, p.519-533, 1988 .

PURVIS, O.N. Studies in vernalisation of cereals. V. The inheritance of the spring and winter habit in hybrids of Petkus rye. Annals of Botany, v.3, n.6, p.419-729, 1939.

Studies in vernalisation of cereals. X. The effect of depletion of carbohydrates on the growth and vernalisation response of excised embryos. Annals of Botany, v.11, n.3, p.269-283, 1947.

Studies in vernalisation of cereals. XI. The effect of date of sowing and of excising the embryo upon the response of Petkus rye to different periods of vernalization treatment. Annals of Botany, v.12, n.2, p.183-206, 1948.

PURVIS, O.N.; GREGORY, F.G. Studies in vernalisation of cereals. I. A comparative study of vernalisation in winter rye by low temperature and by short days. Annals of Botany, v. 1, n.4, p.569-592, 1937.

Studies in vernalisation of cereals. XII. The reversibility by high temperature of the vernalised condition in Petkus winter rye. Annals of Botany, v.16, n.1, p.1-21, 1952.

RAWSON, H.M.; ZAJAC, M.; PENROSE, L.D.J. Effect of seedling temperature and its duration on development of wheat cultivars differing in vernalizing response. Field Crops Research, v.57, n.3, p.289-300, 1998.

REES, A.R. The physiology of ornamental bulbous plants. The Botanical Review, v.32, n.1, p.1-23, 1966.

ROH, M.S.; WILKINS, H.F. The effects of bulb vernalization and shoot photoperiod tratments on growth and flowering of Lilium longiflorum Thunb. Cv. Nellie White Journal of the American Society for Horticultural Science, v.102, n.3, p.229-235, 1977

STRECK, N.A. A generalized nonlinear air temperature response function for node appearance rate in muskmelon (Cucumis melo L.). Revista Brasileira de Agrometeorologia, v.10, n.1, p.105-111, 2002a.

A generalized vernalization response function for lily (Lilium spp.). Revista Brasileira de Agrometeorologia, v.10, n.2, p.221-228, 2002b.

Improving predictions of developmental stages in winter wheat: a modified Wang and Engel model. Agricultural and Forest Meteorology, v.115, p.139-150, 2003.

WANG, S. et al. Vernalization in wheat. I - A model based on the interchangeability of plant age vernalization duration. Field Crops Research, v.41, n.2, p.91-100, 1995.

WANG, S.Y.; ROBERTS, A.N. Physiology of dormancy in Lilium longiflorum 'Ace', Thunb. Journal of the American Society for Horticultural Science, v.95, n.5, p.554-558, 1970 .

WEILER, T.C. Easter lilies. In: LARSON, A. (Eds.). Introduction to floriculture. 2.ed. San Diego : Academic, 1992. 610p.

WEILER, T.C.; LANGHANS, R.W. Determination of vernalizing temperatures in the vernalization requirement of Lilium longiflorum (Thunb.) cv "Ace". Proceedings of the American Society for Horticultural Science, v.93, n.1, p.623-629, 1968.

YAN, W.; HUNT, L.A. Reanalysis of vernalization data of wheat and carrot. Annals of Botany, v.84, n.5, p.615-619, 1999.

ZACHARIUS, R.M.; CATHEY, H.M.; STEWARD, F.C. Nitrogenous compounds and notrogen metabolism in the liliaceae. III. Changes in the soluble nitrogen compounds of the tulip and their relation to flower formation in the bulb. Annals of Botany, v.21, n.82, p.93-201, 1957.

Ciência Rural, v. 34, n.4, jul-ago, 2004. 